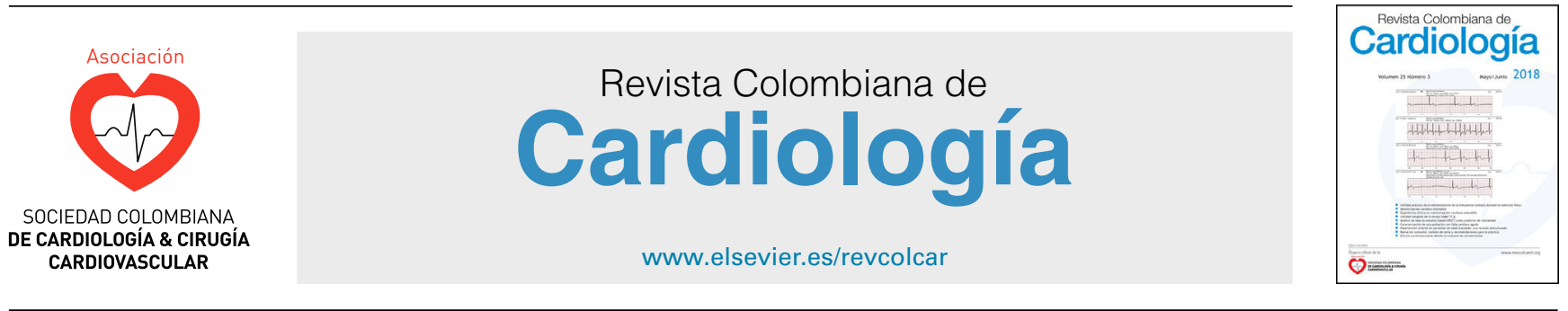

CARDIOLOGÍA DEL ADULTO - REVISIÓN DE TEMAS

\title{
Uso de desfibriladores implantables y terapia de resincronización cardiaca en ancianos mayores de 70 a 80 años: controversias y evidencia
}

\author{
Jainer Méndez-Flórez*, Yessica Agudelo-Zapata, María Carolina Torres Villarreal, \\ Lina Paola-León, Gloria Guarín-Loaiza, Fabio Torres-Saavedra, \\ Álvaro Burgos-Cárdenas y Guillermo Mora-Pabón
}

\author{
Universidad Nacional de Colombia, Bogotá D.C, Colombia
}

Recibido el 19 de noviembre de 2018; aceptado el 11 de febrero de 2019

Disponible en Internet el 12 de julio de 2019

\author{
PALABRAS CLAVE \\ Muerte súbita \\ cardiaca; \\ Terapia de \\ resincronización \\ cardiaca; \\ Desfibriladores \\ implantables; \\ Septuagenarios
}

\begin{abstract}
Resumen Prevenir la muerte súbita y aumentar la supervivencia y la calidad de vida en los pacientes con falla cardiaca son las principales indicaciones para el uso de los desfibriladores implantables y la terapia de resincronización cardiaca respectivamente. Hay certeza de la eficacia clínica de estos dispositivos para la población general; sin embargo, no es tan clara para los pacientes mayores de 70 años dado que esta población no está lo suficientemente representada en los diseños de los estudios clínicos.

La evidencia disponible hasta el momento precisa que en este grupo etario existe beneficio de la terapia de resincronización cardiaca, pero es cuestionable el uso de cardiodesfibriladores. Así mismo, es indispensable tener en cuenta no sólo el riesgo de mortalidad de los pacientes sino también su funcionalidad y fragilidad para definir si se benefician o no de estos dispositivos. (c) 2019 Sociedad Colombiana de Cardiología y Cirugía Cardiovascular. Publicado por Elsevier España, S.L.U. Este es un artículo Open Access bajo la licencia CC BY-NC-ND (http:// creativecommons.org/licenses/by-nc-nd/4.0/).
\end{abstract}

Use of implantable defibrillators and cardiac resynchronisation therapy in patients more than $\mathbf{7 0}$ to $\mathbf{8 0}$ years old: arguments and evidence

\begin{abstract}
The prevention of sudden death, to increase the survival, and quality of life in patients with heart failure, are the main indications for the use of implantable defibrillators and cardiac resynchronisation therapy, respectively. There is confidence in the clinical efficacy of these devices for the general population, but it is not so clear in patients over 70 years-old, given that this population is not sufficiently represented in clinical study designs.
\end{abstract}

\section{KEYWORDS}

Cardiac sudden

death;

Cardiac

resynchronisation

therapy;

Implantable

defibrillators;

Septuagenarians

\footnotetext{
* Autor para correspondencia.

Correo electrónico: jainer87@hotmail.com (J. Méndez-Flórez).
} 
Although the evidence available up until now shows that there is a benefit in this age group for the use cardiac resynchronisation therapy, the use of cardiac defibrillators is questionable. It is also indispensable to not only to take into account the mortality risk in these patients, but also their functionality and frailty in order to determine whether or not they would benefit from these devices.

(c) 2019 Sociedad Colombiana de Cardiología y Cirugía Cardiovascular. Published by Elsevier España, S.L.U. This is an open access article under the CC BY-NC-ND license (http:// creativecommons.org/licenses/by-nc-nd/4.0/).

\section{Introducción}

La proporción de adultos mayores de 70 años viene en aumento y cada vez más se convierte en una población subsidiaria de intervenciones médicas que prolongan la vida. En Colombia se estima que para el año 2050 el grupo mayor de 60 años sea aproximadamente el $20 \%$ de la población total ${ }^{1}$. Con el aumento en la esperanza de vida también se espera que incremente la prevalencia de enfermedades como falla cardiaca y el riesgo de muerte súbita; datos actuales muestran una incidencia en los Estados Unidos de hasta 650.000 casos nuevos de falla cardiaca al año, con una mortalidad del $50 \%$ a los cinco años del diagnóstico ${ }^{2}$. Los pacientes septuagenarios no están bien representados en los ensayos clínicos; en los años setenta el porcentaje de pacientes mayores de 75 años incluidos en los estudios alcanzaba tan solo el $2 \%$, con un incremento al $13 \%$ en los años noventa, cantidad aún insuficiente, teniendo en cuenta el aumento de la proporción de septuagenarios en la sociedad actual ${ }^{3}$.

La edad es un factor de riesgo independiente para el desarrollo de la falla cardiaca. Registros muestran una prevalencia de hasta 80 casos por 1.000 individuos en mayores de 85 años, así como mayor riesgo de hospitalizaciones y muerte por esta condición ${ }^{4}$. La evidencia también indica que la aparición de arritmias ventriculares, específicamente taquicardia ventricular sostenida o no, es más frecuente en pacientes mayores de 65 años con cardiopatía estructural de base, con una prevalencia variable entre 20 y el $80 \% 5$. También, posiblemente relacionado con los datos previos, hay incremento en la presentación de muerte súbita, con registros que van del 8.3 casos por 1.000 personas-año en mayores de 80 años, hasta 14.9 casos por 1.000 personas-año en mayores de 90 años$^{6}$.

Los cardiodesfibriladores implantables (CDI) y la terapia de resincronización cardiaca (TRC) se emplean para la prevención, tanto primaria como secundaria de la muerte súbita y el manejo de la disincronía ventricular en la falla cardiaca, respectivamente. Las indicaciones para el uso de estos dispositivos en la población general están dadas por guías internacionales basadas en la evidencia (tabla 1), sin que en ninguna se especifique un límite de edad ${ }^{7}$.

\section{Generalidades de los dispositivos cardiacos implantables}

Los dispositivos implantables han revolucionado el tratamiento de las enfermedades cardiacas y cada vez su uso
Tabla 1 Indicaciones de cardiodesfibrilador implantable y cardiorresincronizador

\begin{tabular}{|c|c|}
\hline \multicolumn{2}{|l|}{ Cardiodesfibrilador implantable } \\
\hline \multicolumn{2}{|l|}{ Prevención primaria } \\
\hline \multicolumn{2}{|l|}{$\begin{array}{l}\text { Pacientes con fracción de eyección igual o } \\
\text { menor de } 35 \% \text { clase funcional NYHA II a III en } \\
\text { manejo médico óptimo durante al menos tres } \\
\text { meses, expectativa de vida de al menos un año } \\
\text { y buena funcionalidad. }\end{array}$} \\
\hline Cardiopatía isquémica & IA \\
\hline Cardiopatía no isquémica & IB \\
\hline \multicolumn{2}{|l|}{ Prevención secundaria } \\
\hline $\begin{array}{l}\text { Pacientes que presentaron una arritmia } \\
\text { ventricular que causó inestabilidad } \\
\text { hemodinámica, con expectativa de vida mayor } \\
\text { de un año. } \\
\text { Cardiorresincronizador }\end{array}$ & IA \\
\hline $\begin{array}{l}\text { Pacientes con fracción de eyección igual o } \\
\text { menor del } 35 \% \text {, ritmo sinusal, clase funcional II } \\
\text { - IV en manejo médico óptimo por al menos } \\
\text { tres meses, expectativa de vida mayor de un } \\
\text { año y buena funcionalidad. }\end{array}$ & \\
\hline Bloqueo de rama izquierda $\mathrm{QRS}>150 \mathrm{~ms}$ & IA \\
\hline Bloqueo de rama izquierda QRS 130 - $150 \mathrm{~ms}$ & IB \\
\hline Bloqueo de rama derecha $\mathrm{QRS}>150 \mathrm{~ms}$ & IIA \\
\hline Bloqueo de rama derecha QRS 130 - $150 \mathrm{~ms}$ & IIB \\
\hline
\end{tabular}

ha sido extendido a población mayor de 70 años. La primera terapia estimuladora fue el marcapasos, a partir de lo cual se diseñaron nuevos dispositivos que podían reconocer arritmias ventriculares y reestablecer la sincronía eléctrica y mecánica del corazón. Con estos desarrollos nació el cardiodesfibrilador implantable (CDI) y la terapia de resincronización combinada con cardiodesfibrilador (TRC-D) o no $(\text { TRC-P) })^{8,9}$

La evidencia disponible hasta el momento ha demostrado el beneficio de estas terapias en pacientes cuidadosamente seleccionados; sin embargo, el uso de estos dispositivos en ancianos no está claramente definido. Las recomendaciones actuales surgen del análisis de subgrupos con resultados divergentes para el uso de cada dispositivo. Aun así, la edad no se ha considerado criterio de exclusión para el uso de estas terapias ${ }^{10,11}$. 
El CDI permite el reconocimiento de arritmias ventriculares y genera un impulso eléctrico de alta energía una vez se detecta la arritmia. La capacidad del CDI para terminar las arritmias ventriculares ya sea con descarga eléctrica o terapia antitaquicardia, es la característica fundamental de este dispositivo. El beneficio del uso del CDI se ha demostrado en prevención primaria y secundaria para muerte súbita ${ }^{12,13}$.

El TRC es útil para el manejo de la disincronía eléctrica y mecánica ventricular. La evidencia actual demuestra el beneficio de esta terapia en la disminución de la mortalidad y de hospitalizaciones por falla cardiaca, además de su impacto en otros aspectos como calidad de vida y tolerancia al ejercicio $8,12,14,15$. Sin embargo, es importante recodar que aproximadamente $30 \%$ de los pacientes no responden a la TRC.

\section{Cardiodesfibrilador implantable en el paciente mayor de 70 años}

Los estudios de eficacia clínica de CDI han excluido en gran parte la población mayor de 70 años y sólo el $20 \%$ de los pacientes fueron mayores de 75 años, por lo que no es posible tener evidencia y recomendaciones de calidad en este subgrupo ${ }^{16}$.

Algunos autores señalan que el CDI no tiene un impacto en la mejoría de la calidad de vida e incluso podría empeorarla, principalmente debido a la frecuencia con que suceden descargas inapropiadas (hasta en un $20-25 \%$ en el seguimiento a 45 meses $)^{17}$. Pese a ello, con la optimización de las terapias actuales el porcentaje de descargas inapropiadas es mucho menor. Estos datos contrastan con la información encontrada para la TRC-D, en los que estos dispositivos disminuyen las descargas inapropiadas respecto al CDI y podrían ser más eficaces en esta población con alta carga de comorbilidades ${ }^{18}$.

En prevención primaria de muerte súbita, el uso de CDI en pacientes mayores de 70 años no isquémicos es discutido puesto que no impactan la mortalidad general, aunque sí disminuyen la mortalidad por muerte súbita ${ }^{19,20}$. En el estudio DANISH se evaluó la eficacia de los cardiodesfibriladores en pacientes con cardiopatía no isquémica como prevención primaria, y demostró que su uso no disminuye la mortalidad global en esta población; en el análisis por subgrupos la población mayor de 68 años es la que menos se beneficia de estos dispositivos (HR 1.19 IC 95\% 0.81-1.73 p 0.38) ${ }^{21}$. No obstante, es importante señalar que en dicho estudio un tercio de los pacientes tenían más de 68 años y la mortalidad por todas las causas superó a la mortalidad cardiovascular, lo cual podría disminuir el impacto del CDI en esta población teniendo en cuenta los riesgos competitivos que condicionan la supervivencia en este grupo. Por tanto sugerimos que, en esta etiología en particular se deben tener en cuenta las comorbilidades y la calidad de vida de los pacientes para definir si se benefician o no del uso de CDI. Exposito et al. demostraron como los pacientes mayores de 75 años con CDI tienen mayor mortalidad no cardiovascular y mortalidad por todas las causas que la población menor de 75 años, dado que este grupo poblacional tiene mayor número de comorbilidades, como enfermedad pulmonar obstructiva crónica, enfermedad renal crónica e hipertensión, que condicionan su sobrevida ${ }^{22}$.
En prevención secundaria los CDI han mostrado datos más consistentes en cuanto a disminución de la mortalidad ${ }^{23}$. Estudios observacionales muestran que la edad por sí sola no es un factor limitante para definir la implantación del dispositivo. Posiblemente sea más relevante la funcionalidad previa del paciente ${ }^{24}$. En este último punto hacen énfasis algunos documentos internacionales que tratan de definir qué pacientes mayores de 75 años pueden beneficiarse de implante de CDI. Las recomendaciones al respecto van ligadas a evaluar la funcionalidad del paciente teniendo en cuenta dependencia funcional, condición cognitiva, comorbilidades, presencia o no de síndromes geriátricos, número de hospitalizaciones y soporte social, antes de implantar el dispositivo ${ }^{25}$.

Cabe señalar también que las complicaciones relacionadas con la implantación de CDI no tienen relación con la edad del paciente. El neumotórax, el hemotórax, el accidente cerebrovascular o la muerte relacionados con el procedimiento, son similares en pacientes mayores y menores de 75 años ${ }^{26}$.

\section{TRC-P en mayores de 70 años}

La terapia de resincronización cardiaca ha demostrado disminuir la mortalidad y mejorar los síntomas, la calidad de vida, la función ventricular y la capacidad de ejercicio en pacientes con falla cardiaca con fracción de eyección reducida y QRS mayor de 130 milisegundos; sin embargo, la evidencia es de menor calidad en otros grupos como los pacientes con fibrilación auricular permanente que no fueron incluidos en los grandes estudios aleatorizados ${ }^{27,28}$.

En los estudios COMPANION y CARE - HF se incluyeron pacientes con clase funcional III - IV con FEVI $\leq 35 \%$ y duración del $\mathrm{QRS} \geq 120 \mathrm{~ms}$, con un desenlace primario de muerte y hospitalizaciones. Se comparó el uso de TRC con la terapia médica óptima, y se demostró que reducir la mortalidad en $34 \%$ y las hospitalizaciones en un $39 \% 27,28$. Por otra parte, en el estudio REVERSE se incluyeron pacientes menos comprometidos con clases funcionales I - II, $\mathrm{FEVI} \leq 40 \%$ y duración del $\mathrm{QRS} \geq 120 \mathrm{~ms}$, y se demostró menor deterioro de la clase funcional en un $16 \%$ y mejoría en medidas de remodelación del ventrículo izquierdo, sin disminución de la mortalidad estadísticamente significativa ${ }^{29}$. Con los datos de estos y otros estudios se han fundamentado las recomendaciones de uso de TRC (tabla 1).

La evidencia en pacientes ancianos es menos robusta. Delnoy et al. evaluaron 266 pacientes, 39 de los cuales eran octogenarios; midieron parámetros ecocardiográficos y clínicos al inicio del estudio y hasta los 24 meses, y evidenciaron mejoría significativa en la clase funcional, la fracción de eyección basal y la respuesta clínica durante el seguimiento sin diferencias en los ancianos ${ }^{30}$. En dos estudios de cohorte, uno que incluyó 26.887 pacientes y otro 220 , con mayores de 80 años que representaron el $17,5 \%(n=4.694)$ en la primera y $22,2 \%(n=49)$ en la segunda, se encontraron tasas de mortalidad más altas en los octogenarios en comparación con los pacientes más jóvenes ${ }^{19,31}$.

Sin embargo, no todos los estudios muestran mayor mortalidad en ancianos; Kelli et al. compararon pacientes mayores de 80 años con menores de esta edad, usuarios de estos dispositivos, y no evidenciaron diferencias 
significativas en la tasa de mortalidad por cualquier causa a los 36 meses, la cual fue del 11 y $8 \%$, respectivamente ( $p$ $0,381)^{32}$.

Cuando se evalúan los resultados de la TRC-P contra el manejo médico óptimo, estos son alentadores en esta población. Rickard et al. compararon octogenarios con falla cardiaca en TRC-P con pacientes sin dispositivos, y mostraron una supervivencia a 2 años de $78,9 \%$ en los pacientes con dispositivo, con divergencia de las curvas de mortalidad post implante respecto al grupo control $(p 0,03)^{33}$.

Igualmente, la terapia de resincronización cardiaca ha mostrado utilidad en cuanto a disminución de síntomas y aumento en la capacidad de ejercicio; incluso se ha demostrado como el TRC desempeña un papel crucial ya que mejora el estado funcional y cognitivo de los pacientes, efecto que tiene mayor impacto en población octogenaria ${ }^{34}$.

\section{Terapia de resincronización cardiaca combinada con cardiodesfibrilador}

La TRC-D pretende mejorar la disincronía electromecánica ventricular que hace parte de la fisiopatología de la ICC, y además disminuir el riesgo de muerte súbita a través del CDI incorporado ${ }^{35}$. La implantación de este dispositivo ha aumentado entre los mayores de 75 años; sin embargo, la evidencia del uso de TCR-D en esta población se basa en análisis de subgrupos de estudios como el COMPANION en el que los sujetos mayores de 65 años tenían mayor beneficio de la TRC-D ${ }^{8,27}$. Otros análisis por subgrupos son proporcionados por los estudios RAFT, MADIT-CRT, y REVERSE. De estos el RAFT y el REVERSE no mostraron beneficio para los mayores de 65 años. En el REVERSE se comparó un desenlace compuesto en el que se medía clase funcional, hospitalización y muertes por falla cardiaca, entre un grupo con el TCR-D encendido y otro grupo con el dispositivo apagado, sin que se encontrara ninguna diferencia estadísticamente significativa ${ }^{36}$. No obstante, en el MADIT-CRT, la TRC-D redujo significativamente la variable combinada: muerte o empeoramiento de falla cardiaca en los mayores de 75 años ${ }^{37}$.

Delnoy et al. evaluaron los resultados clínicos y ecocardiográficos de pacientes menores y mayores de 70 años, y evidenciaron que ambos grupos mostraron mejoría similar en la clase NYHA, calidad de vida y fracción de eyección del ventrículo izquierdo durante dos años de seguimiento ${ }^{30}$. Otro estudio similar determinó la respuesta clínica y ecocardiográfica a la TRC durante más de dos años. En general, los resultados en menores y mayores de 75 años son comparables en cuanto a respuesta clínica, incluida la calidad de vida y la distancia recorrida en 6 minutos ${ }^{38}$.

De la Cruz et al. compararon TRC frente a la terapia médica (TM) y sugirieron que es potencialmente beneficiosa en términos de mortalidad en los mayores de 75 años $^{39}$; sin embargo, cuando se comparó TCR con o sin CDI, estudios recientes no demostraron ningún beneficio en mortalidad por adicionar el CDI a una terapia de resincronización cardiaca (HR 1.16; 95\% IC, 0.70 - 1.92; p 0.563) ${ }^{40}$.

Por su parte, Kubala et al. valoraron el impacto del estado de fragilidad sobre la respuesta a la terapia de resincronización en pacientes mayores de 70 años y determinaron que la fragilidad, definida como un puntaje menor de 14 en el
ONCODAGE G8 Score ${ }^{41}$, es un predictor fuerte de respuesta a este tipo de dispositivos, dado que los pacientes más frágiles tienen mayor riesgo de mortalidad, hospitalizaciones por falla cardiaca y posibilidad de no aumentar la fracción de eyección, lo cual sugiere que esta escala es un buen método para definir si un paciente se beneficiará o no del implante de $\mathrm{CRT}^{42}$ (tabla 2). En contraste con lo anterior, hasta la fecha no hay estudios de validación de escalas de fragilidad para definir si los pacientes mayores de 70 años se podrían o no beneficiar del implante de un cardiodesfibrilador.

Las descargas del CDI producen dolor y disminución de la calidad de vida. En pacientes ancianos, ante situaciones de fin de vida por su enfermedad cardiovascular u otras (cáncer, demencia...), es importante definir con ellos y sus familias la utilidad o no del mantenimiento de terapias para tratar eventos de arritmias ventriculares sostenidas. Es evidente que los dispositivos cardiacos implantables hacen parte del manejo médico de enfermedades cardiovasculares y debe reconocerse que en estadios de final de vida es razonable desistir de la terapia médica con el objetivo de acortar el sufrimiento del paciente, dado que no se logrará impactar

Tabla 2 Oncodage G8 Score

\begin{tabular}{|c|c|}
\hline Oncodage g8 Score & \\
\hline Pérdida de peso en los últimos tres meses & \\
\hline$>3 \mathrm{~kg}$ & 0 \\
\hline No sabe & 1 \\
\hline $1-3 \mathrm{~kg}$ & 2 \\
\hline No pérdida de peso & 3 \\
\hline Movilidad & \\
\hline Cama o silla & 0 \\
\hline Capacidad de levantarse pero no caminar & 1 \\
\hline Caminar & 2 \\
\hline Problemas neuropsicológicos & \\
\hline Demencia o depresión severa & 0 \\
\hline Demencia o depresión leve & 1 \\
\hline No problemas neuropsicológicos & 3 \\
\hline Índice de masa corporal $\left(\mathrm{kg} / \mathrm{m}^{2}\right)$ & \\
\hline$<18,5$ & 0 \\
\hline $18,5 \leq 21$ & 1 \\
\hline $21 \leq 23$ & 2 \\
\hline$>2 \overline{3}$ & 3 \\
\hline ¿Toma más de tres medicamentos diarios? & \\
\hline Sí & 0 \\
\hline No & 1 \\
\hline ¿Cómo considera su estado de & \\
\hline salud en comparación con & \\
\hline otras personas de su edad? & \\
\hline No $\tan$ bueno & 0 \\
\hline No sabe & 0,5 \\
\hline Igual & 1 \\
\hline Mejor & 2 \\
\hline Edad (años) & \\
\hline$>85$ & 0 \\
\hline $80-85$ & 1 \\
\hline$<80$ & 2 \\
\hline
\end{tabular}




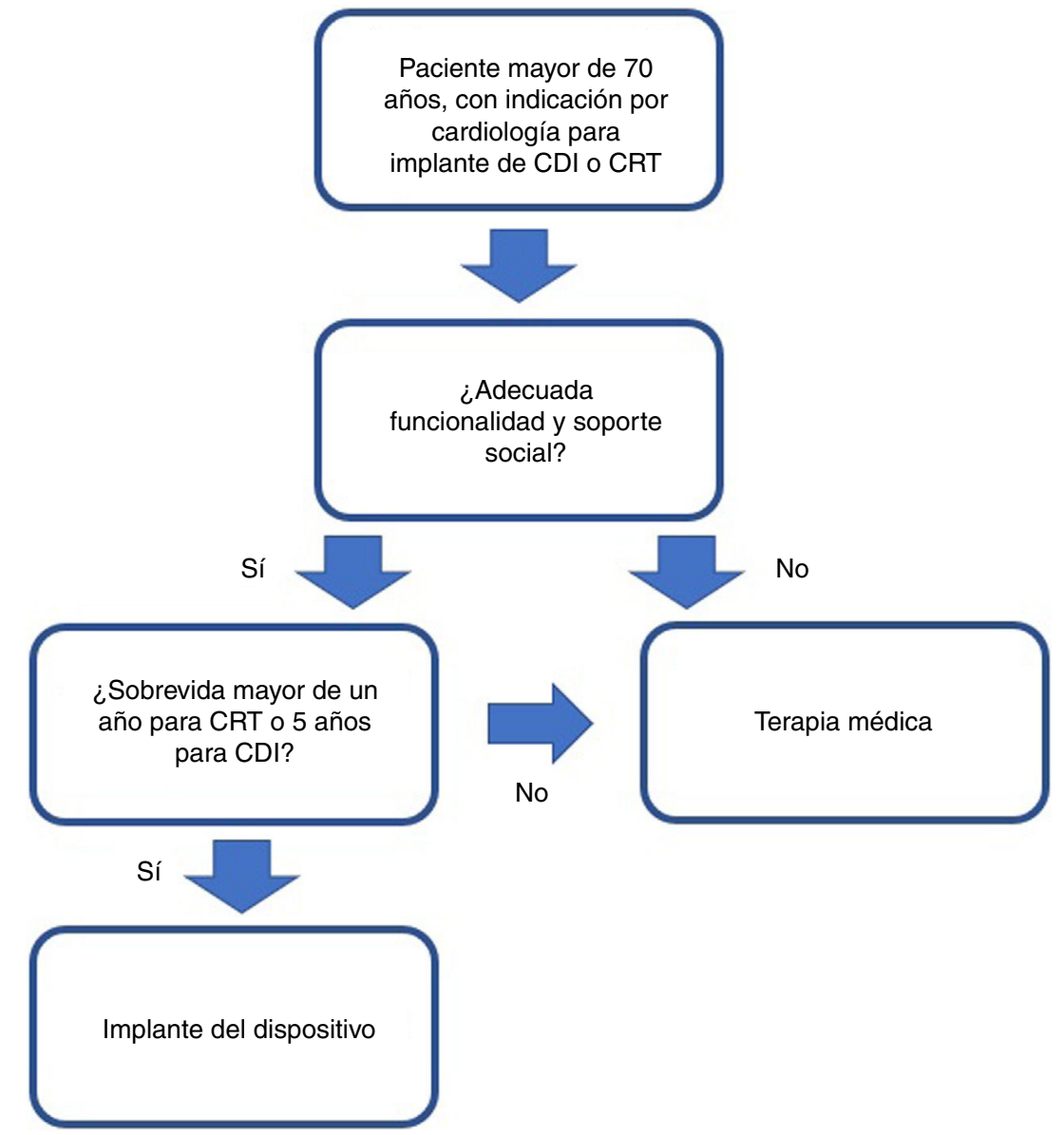

Figura 1 Algoritmo sugerido para el implante de cardiodesfibriladores o dispositivos de terapia de resincronización cardiaca en mayores de 70 años.

en la sobrevida con calidad a largo plazo. Por ello la desactivación de terapias del CDI hace parte del manejo compasivo de estos pacientes y no significa ninguna falta a la ética médica ${ }^{43}$.

\section{Costo-efectividad de los dispositivos}

El análisis de costo-efectividad evalúa los beneficios y costos de una intervención propuesta comparada con la intervención convencional, para tomar decisiones en salud pública.

Estudios en Estados Unidos muestran que la implantación de un CDI en pacientes de cualquier edad para la prevención primaria de muerte súbita tiene un índice de costo-efectividad menor de 100.000 dólares en comparación con los años de calidad de vida ganados. El beneficio es mayor cuando los pacientes cumplen los criterios de inclusión de los grandes ensayos aleatorizados ${ }^{44}$. La relación de costo-efectividad depende de factores como duración de la batería y electrodos del dispositivo y de riesgo de muerte súbita $^{45}$.

No existen estudios diseñados exclusivamente para evaluar la efectividad en ancianos, por lo que, los análisis de costo-efectividad se realizan a partir de subgrupos. De acuerdo con un metaanálisis en el que se incluyeron pacientes mayores de 75 años se encontró que la terapia con CDI, en este grupo de pacientes, es igual de costoefectiva que en los pacientes de 65 a 74 años $^{46}$. En un subanálisis del grupo de pacientes mayores de 65 del estudio MADIT-II, se demostró que la utilización del dispositivo debe ser por lo menos de 3.4 años, para que sea costoefectivo ${ }^{47}$. Otros estudios más recientes sugieren que el CDI puede llegar a considerarse costo-efectivo en la población adulta mayor con expectativas de vida entre 5 a 7 años $^{48}$.

La TRC con o sin desfibrilador, es costo-efectiva en la población general, tanto para los países de altos y bajos ingresos. Sin embargo, la relación entre años de vida ajustados por calidad con la terapia de resincronización cardiaca es significativamente más costosa en pacientes con desfibrilador implantable. Aunque no se analiza la influencia de la edad en el análisis de costo-efectividad, los autores sugieren que la expectativa de vida de los pacientes se relaciona indirectamente con el costo ${ }^{49}$.

\section{Conclusiones}

La decisión de utilizar TRC o CDI en ancianos mayores de 70-80 años no debe fundamentarse solo en la disminución de la mortalidad. En este grupo de pacientes es tanto o más 
importante evaluar desenlaces relacionados con la calidad de vida. Por ello es necesario realizar una valoración multidimensional en la que se documente el estado funcional del paciente, la fragilidad, las comorbilidades crónicas, el deterioro cognitivo, el estado nutricional, la sarcopenia, el riesgo de caídas y el soporte social antes del implante del CDI o CRT.

Esta revisión plantea que no hay estudios bien diseñados para esta población, que demuestren una eficacia clínica contundente. La discusión continúa, pero por el momento las guías de manejo dejan abierta la posibilidad de que el paciente geriátrico con adecuada funcionalidad sea subsidiario de este tipo terapias. Dada la controversia se presenta un algoritmo que trata de integrar los aspectos de la valoración geriátrica multidimensional y las indicaciones a la luz de las actuales guías para estos dispositivos (fig. 1).

\section{Financiación}

Ninguna.

\section{Conflicto de interés}

Ninguno.

\section{Bibliografía}

1. DANE. Censo de población y vivienda Colombia 2005. [Internet]. Disponible en: https://www.dane.gov.co/index.php/ estadisticas-por-tema/demografia-y-poblacion/censo-general2005-1.

2. Butler J, Casey D, Yancy C, Jessup M, Bozkurt B, Colvin M, et al. 2016 ACC/AHA/HFSA Focused Update on New Pharmacological Therapy for Heart Failure: An Update of the 2013 ACCF/AHA Guideline for the Management of Heart Failure. Circulation. 2016;134:e282-93.

3. Ezekowitz J, Kaul P. The epidemiology and management of elderly patients with myocardial infarction or heart failure. Heart Fail Rev. 2010;15:407-13.

4. Metra M, Cotter G, El-Khorazaty J, Davison B, Milo O, Carubelli $\mathrm{V}$, et al. Acute heart failure in the elderly: differences in clinical characteristics, outcomes, and prognostic factors in the VERITAS study. J Card Fail. 2015;21:179-88.

5. Tresch D. Evaluation and management of cardiac arrhythmias in the elderly. Card Arrhythm. 2001;85:527-49.

6. Niemeijer M, van den Berg M, Leening M, Hofman A, Franco O, Deckers J, et al. Declining incidence of sudden cardiac death from 1990-2010 in a general middle-aged and elderly population: The Rotterdam Study. Hear Rhythm. 2015;12:123-9.

7. Ponikowki P, Voors A, Anker S, Falk V, Bueno H, Cleland J, et al. 2016 ESC Guidelines for the diagnosis and treatment of acute and chronic heart failure. Eur Heart J. 2016;37:2129-200.

8. Kramer D, Reynolds M, Mitchell S. Resynchronization: Considering device-based cardiac therapy in older adults. J Am Geriatr Soc. 2013;61:615-21.

9. Merriam J, Rajendra A, Gold M. Newer indications for ICD and CRT. Cardiol Clin. 2014;32:181-90.

10. Dotsenko O, Barsheshet A, Huang D. Cardiac resynchronization therapy for prevention of heart failure events in elderly patients with left ventricular dysfunction. Expert Rev Cardiovasc Ther. 2012;10:1319-27.

11. Epstein A, Kay G, Plumb V, McElderry H, Doppalapudi H, Yamada $\mathrm{T}$, et al. Implantable cardioverter-defibrillator prescription in the elderly. Hear Rhythm. 2009;6:1136-43.
12. Tung $P$, Albert $C M$. Causes and prevention of sudden cardiac death in the elderly. Nat Rev Cardiol. 2013;10:135-42.

13. Dimarco JP, Mower M. Implantable cardioverter-defibrillators. N Engl J Med. 2003;349:1836-47.

14. Brignole M, Auricchio A, Barón-Esquivias G, Bordachar P, Boriani G, Breithardt O, et al. Guía de práctica clínica de la ESC 2013 sobre estimulación cardiaca y terapia de resincronización cardiaca. Rev Esp Cardiol. 2014;67:e1-60.

15. Lampert R. Quality of Life and End-Of-Life Issues for Older Patients with Implanted Cardiac Rhythm Devices. Clin Geriatr Med. 2012;28:693-702.

16. Huang D, Sesselberg H, McNitt S, Noyes K, Andrews M, Hall W, et al. Improved survival associated with prophylactic implantable defibrillators in elderly patients with prior myocardial infarction and depressed ventricular function?: a MADIT-II substudy. J Cardiovasc Electrophysiol. 2007;18:833-8.

17. Borne RT, Varosy P, Masoudi F. Implantable cardioverterdefibrillator shocks: Epidemiology, outcomes and therapeutic approaches. JAMA Inter Med. 2013;173:859-65.

18. Verbrugge F, Dupont M, De Vusser P, Rivero M, Van Herendael $\mathrm{H}$, Vercammen $\mathrm{J}$, et al. Response to cardiac resynchronization therapy in elderly patients ( $\geq 70$ years) and octogenarians. Eur J Heart Fail. 2013;15:203-10.

19. Suleiman M, Goldenberg IH, Haim M, Schliamser J, Boulos M, Ilan $M$, et al. Clinical characteristics and outcomes of elderly patients treated with an implantable cardioverter-defibrillator or cardiac resynchronization therapy in a real-world setting: Data from the Israeli ICD Registry. Hear Rhythm. 2014;11:435-41.

20. Santangeli P, Di Biase L, Dello Russo A, Casella M, Bartoletti S, Santarelli P, et al. Meta-analysis: age and effectiveness of prophylactic implantable cardioverter-defibrillators. Ann Intern Med. 2010;153:592-9.

21. Kober L, Thune J, Nielsen J, Haarbo J, Korup E, Jensen G, et al. Defibrillator Implantation in Patients with Nonischemic Systolic Heart Failure. N Engl J Med. 2016;375:1221-30.

22. Exposito V, Rodríguez M, González S, Arias M, Sánchez J, La Huerta A, et al. Primary prevention implantable cardioverter defibrillator and cardiac resynchronization therapy-defibrillator in elderly patients: results of a Spanish multicentre study. Europace. 2016;18:1203-10.

23. The Antiarrhythmics Investigators versus ID (AVID). A comparison of antiarrhythmic-drug therapy with implantable defibrillators in patients resuscitated from near-fatal ventricular arrhythmias. N Engl J Med. 1997;337:576-83.

24. Killu A, Wu J, Friedman P, Shen W, Webster T, Brooke K, et al. Outcomes of cardiac resynchronization therapy in the elderly. Pacing Clin Electrophysiol. 2012;36:664-72.

25. Fauchiera L, Alonso C, Anselmec F, Blangyd H, Bordachare P, Boveda S, et al. Position paper for management of elderly patients with pacemakers and implantable cardiac defibrillators: Groupe de Rythmologie et Stimulation Cardiaque de la Société Française de Cardiologie and Société Francaise de Gériatrie et Gérontologie. Arch Cardiovasc Dis. 2016;109:563-85.

26. Eccleston D, Swale M, Phillips K, Tan I, Young G. Is there an Outcome Hazard for Elderly Australians After Device Implantation: Observations from the Genesis Care Cardiovascular Outcomes Registry (GCOR). Heart Lung Circ. 2018;27: S152-3.

27. Bristow M, Saxon L, Boehmer J, Krueger S, Kass D, De Marco T, et al. Cardiac-resynchronization therapy with or without an implantable defibrillator in advanced chronic heart failure. N Engl J Med. 2004;350:2140-50.

28. Cleland J, Daubert J, Erdmann E, Freemantle N, Gras D, Kappenberger $L$, et al. The effect of cardiac resynchronization on morbidity and mortality in heart failure. N Engl J Med. 2005;352:1539-49. 
29. Linde C, Gold M, Abraham W, St John Sutton M, Ghio S, Cerkvenik $J$, et al. Long-term impact of cardiac resynchronization therapy in mild heart failure: 5-year results from the resynchronization reverses remodeling in systolic left ventricular dysfunction (REVERSE) study. Eur Heart J. 2013;34:2592-9.

30. Delnoy P, Ottervanger J, Luttikhuis H, Elvan A, Misier A, Beukema W, et al. Clinical response of cardiac resynchronization therapy in the elderly. Am Heart J. 2008;155:746-51.

31. Swindle J, Rich M, McCann P, Burroughs T, Hauptman PJ. Implantable cardiac device procedures in older patients: use and in-hospital outcomes. Arch Intern Med. 2010;170:631-7.

32. Kelli H, Merchant F, Mengistu A, Casey M, Hoskins M, El-Chami M. Intermediate-term mortality and incidence of ICD therapy in octogenarians after cardiac resynchronization therapy. J Geriatr Cardiol. 2014;11:180-4.

33. Rickard J, Cheng A, Spragg D, Green A, Leff B, Tang W, et al. Survival in octogenarians undergoing cardiac resynchronization therapy compared to the general population. Pacing Clin Electrophysiol. 2014;37:740-4.

34. Fumagalli S, Pieragnoli P, Ricciardi G, Mascia G, Mascia F, Michelotti F, et al. Cardiac resynchronization therapy improves functional status and cognition. Int J Cardiol. 2016;219:212-7.

35. Banchs J, Davinson W, González M. Terapia de Resincronización Ventricular?: ¿es todavía el electrocardiograma el mejor método para la selección de pacientes? Rev Electro y Arritmias. 2010;3:20-31.

36. Daubert C, Gold M, Abraham W, Ghio S, Hassager C, Goode $G$, et al. Prevention of Disease Progression by Cardiac Resynchronization Therapy in Patients With Asymptomatic or Mildly Symptomatic Left Ventricular Dysfunction. J Am Coll Cardiol. 2009;54:1837-46.

37. Penn J, Goldenberg I, Moss A, McNitt S, Zareba W, Klein H, et al. Improved outcome with preventive cardiac resynchronization therapy in the elderly: a MADIT-CRT substudy. J Cardiovasc Electrophysiol. 2011;22:892-7.

38. Bleeker G, Schalij M, Molhoek S, Boersma E, Steendijk P, van der Wall E, et al. Comparison of effectiveness of cardiac resynchronization therapy in patients $<70$ versus $>$ or $=70$ years of age. Am J Cardiol. 2005;96:420-2.

39. De la Cruz E, Cortés M, Farré J, Palfy J, Ávila P, Hernández I, et al. Comparison of pharmacological treatment alone versus treatment combined with cardiac resynchronization therapy in patients over 75 years. J Interv Card Electrophysiol. 2015;43:13-20.

40. Doring M, Eber M, Dagres N, Mussigbrodt A, Bode K, Knopp K, et al. Cardiac resynchronization therapy in the ageing population - With or without an implantable defibrillator? Int J Cardiol. 2018;263:48-53.

41. Hamaker M, Jonker J, de Rooij S, Vos A, Smorenburg C, van Munster B. Frailty screening methods for predicting outcome of a comprehensive geriatric assessment in elderly patients with cancer: a systematic review. Lancet Oncol. 2012;13: e437-44.

42. Kubala M, Guedon L, Anselme F, Klug D, Bertaina G, Traulle S, et al. Utility of Frailty Assessment for Elderly Patients Undergoing Cardiac Resynchronization Therapy. J Am Coll Cardiol. EP. 2017:3

43. Padeletti L, Arnar D, Boncinelli L, Brachman J, Camm J, Daubert J, Hassam S, et al. EHRA Expert Consensus Statement on the management of cardiovascular implantable electronic devices in patients nearing end of life or requesting withdrawal of therapy. Europace. 2010;12:1480-9.

44. Sanders G, Hlatky M, Owens D. Cost-Effectiveness of Implantable Cardioverter-Defibrillators. N Engl J Med. 2005;353:1471-80.

45. Gialama F, Prezerakos P, Maniadakis N. The cost effectiveness of implantable cardioverter defibrillators: A systematic review of economic evaluations. Appl Health Econ Health Policy. 2014;12:41-9.

46. Sanders G, Kong M, Al-Khatib S, Peterson E. Cost-effectiveness of implantable cardioverter defibrillators in patients $\geq 65$ years of age. Am Heart J. 2010;160:122-31.

47. Pellegrini C, Lee K, Olgin J, Turakhia M, Tseng Z, Lee R, et al. Impact of advanced age on survival in patients with implantable cardioverter defibrillators. Europace. 2008;10:1296-301.

48. Callejo D, Guerra M, Hernandez-Madrid A, Blasco J. Economic assessment of cardiac resynchronization therapy. Rev Esp Cardiol. 2010;63:1235-43.

49. Bertoldi E, Rohde L, Zimerman L, Pimentel M, Polanczyk C. Cost-effectiveness of cardiac resynchronization therapy in patients with heart failure: The perspective of a middleincome country's public health system. Int J Cardiol. 2013;163: 309-15. 